\section{Desigualdades de gênero no ensino superior e no mercado de trabalho no Brasil: uma análise de idade, período e coorte}

\author{
Carlos Antonio Costa Ribeiro*
}

\begin{abstract}
Resumo: $\mathrm{O}$ artigo analisa o acesso diferencial de homens e mulheres ao ensino superior e a entrada no mercado de trabalho dos diplomados no Brasil entre 1981 e 2006 . Tendo em vista que esses indivíduos nasceram entre 1918 e 1981, porque tinham entre 25 e 64 anos quando foram entrevistados, as análises levam em conta não apenas o período que se inicia em 1981, mas também o efeito das coortes de nascimento que se iniciam em 1918. Para verificar os efeitos de período, coorte e idade utilizo modelos que permitem distinguir estes três efeitos temporais. O principal objetivo é analisar em que medida os ciclos de desenvolvimento e expansão econômica (até a segunda metade da década de 1970) e de estagnação e crise nas décadas de 1980 e 1990 são concomitantes às tendências populacionais de acesso à universidade e de entrada no mercado de trabalhos dos indivíduos com formação universitária. O artigo conclui que há efeitos de coorte e de período bem como diminuição das desigualdades de gênero.
\end{abstract}

Palavras-chave: desigualdade de gênero; educação superior; mercado de trabalho; metodologia para idade, período e coorte.

\section{Introdução}

$\square$

m que medida os ciclos econômicos de desenvolvimento e crise, que marcaram a história das últimas décadas, se relacionam às tendências populacionais de acesso à universidade? Em que medida a população de trabalhadores com nível superior teve acesso diferenciado a posições ocupacionais no topo da hierarquia ao longo das últimas décadas? Neste trabalho, procuro investigar essas questões a partir da análise de séries temporais levando em conta o período de 1981 a 2006, as coortes de idade nascidas entre 1918 e 1981, e os grupos de idade entre 25 e 64 anos. As perguntas não são novas, mas acredito que a forma de as abordar pode trazer contribuição para o que já conhecemos sobre o assunto. Nas últimas décadas, o Brasil passou por momentos marcantes de expansão e crise econômica. De forma sumária podemos dividir a história econômica brasileira recente em dois grandes períodos: um primeiro, nas décadas de 1960 e 1970, de forte crescimento econômico; e outro, nas décadas de 1980 a 1990, de estagnação econômica. Só recentemente, nos anos 2000, houve sinais de recuperação do crescimento, tendência que começou a se reverter no final daquela década. Embora as análises apresentadas não permitam estabelecer relações de causalidade, elas possibilitam descrever as tendências popu-

\footnotetext{
* Professor de sociologia no Instituto de Estudos Sociais e Políticos da Universidade Estadual do Rio de Janeiro (lesp-Uerj). $<$ carloscr@iesp.uerj. br>.
} 
lacionais de acesso à universidade e às ocupações de profissionais e administradores de alto nível. Ao interpretar essas tendências populacionais, no entanto, procuro relacioná-las, mesmo se apenas de forma sugestiva, à expansão educacional e às flutuações macroeconômicas que caracterizaram a história recente do país.

Para entender as possíveis relações entre as flutuações macroeconômicas, as condições de escolaridade e a inserção no mercado de trabalho, argumento que é essencial distinguir dois efeitos temporais distintos. Por um lado, as condições de escolaridade e trabalho podem estar relacionadas ao momento do ciclo de vida em que as pessoas estão entrando na universidade ou no mercado de trabalho. Tendências em séries temporais de escolaridade e trabalho relacionadas ao ciclo de vida expressam-se através de um efeito que pode ser observado ao longo das diferentes coortes de nascimento. Por exemplo, uma coorte que esteja no momento de entrar na universidade quando há uma crise econômica pode ter suas oportunidades de avanço educacional minadas, na medida em que um grande número de pessoas decide abandonar os estudos para se dedicar ao trabalho. Assim, para descrever e interpretar as tendências de escolaridade e trabalho ao longo das coortes de nascimento, é importante levarmos em conta o contexto e a conjuntura econômica em que viveram estas coortes. Por outro lado, pode haver mudanças macroeconômicas que estejam relacionadas não apenas às coortes, mas à toda a força de trabalho de uma sociedade. Por exemplo, uma crise econômica pode levar a um aumento do desemprego ou a uma diminuição do acesso a ocupações de nível profissional para todas as pessoas em um determinado período, independentemente do momento em que entraram no mercado de trabalho. Assim, flutuações macroeconômicas podem ser vivenciadas por apenas algumas coortes de idade (efeitos que por vezes duram por toda a vida daquelas coortes), mas também podem afetar a vida de toda a população economicamente ativa em um determinado período (um ou mais anos). No primeiro caso, observaríamos efeitos de coortes de nascimento e, no segundo, efeitos de períodos históricos. Distinguir estas duas tendências históricas é fundamental para entender as consequências das flutuações econômicas nas oportunidades de vida das pessoas. Para verificar a presença de tais tendências históricas, também é necessário levar em conta as mudanças que ocorrem ao longo da vida de uma mesma pessoa, ou seja, mudanças que ocorrem com o envelhecimento e, portanto, que se expressam nos diferentes grupos de idade. A renda, por exemplo, tende a aumentar até uma certa idade e depois se estabiliza ou decresce.

Em suma, para entender e analisar as mudanças históricas nas tendências de escolaridade e trabalho é essencial levar em conta três efeitos distintos: período, coorte e idade. Na realidade, estudos que levem em conta apenas um dos três efeitos podem chegar a resultados incorretos, caso haja influência de alguma das duas outras características. Portanto, o objetivo desta pesquisa é justamente apresentar as ten- 
dências estimadas para mudanças ao longo das coortes de nascimento, dos grupos de idade e do período, ou seja, as tendências levando em conta o possível efeito de cada um dos três fatores. Para analisar as séries históricas, estimo modelos de idade, período e coorte recentemente desenvolvidos por Yang, Fu e Land (2004) e Yang, Schulhofer-Wohl, Fu e Land (2008) ${ }^{1}$. Além disso, lanço mão de gráficos comparando as taxas brutas (observadas simplesmente pelas estatísticas descritivas) e as taxas ponderadas (baseadas nos modelos estimados) ao longo dos grupos de idade, das coortes de nascimento e dos anos das Pesquisas Nacionais de Amostra de Domicílios (Pnad). Se os modelos indicarem que há um efeito de coorte, de idade e/ou de período, devemos entender as taxas estimadas como correções das taxas brutas. Ou seja, quando os modelos se ajustam bem aos dados as taxas estimadas corrigem as brutas porque levam em conta o efeito de cada um dos fatores nos outros. Por exemplo, a taxa estimada de participação na universidade ao longo das coortes controla os efeitos de idade e período, e assim por diante.

Estudos anteriores indicam que os níveis educacionais da população estão fortemente relacionados a tendências históricas que se expressam por meio das coortes de nascimento (Breen \& Jonsson, 2005; 2007; Shavit \& Blossfeld, 1993; Ribeiro, 2009). Tendo em vista que a participação no sistema educacional está correlacionada à idade das pessoas, é normal que efeitos de mudanças históricas nas oportunidades de acesso e progressão no sistema educacional se expressem para diferentes coortes de nascimento. Por exemplo, pessoas nascidas em 1940 estavam na idade de entrar na escola em 1946, quando o sistema educacional brasileiro ainda era incipiente. Ao passo que pessoas que nasceram em 1980 estavam na idade de entrar na escola em 1986, quando se iniciou a expansão do sistema de educação fundamental no Brasil. Desta forma, pessoas nascidas em 1980 têm mais chances de obter educação básica do que pessoas nascidas em 1940. De fato, vários estudos comprovam que mudanças históricas afetam o nível educacional das pessoas a partir de efeitos de coorte (Shavit \& Blonsfeld, 1993). Em contrapartida, mudanças econômicas drásticas (crises ou crescimentos rápidos) podem influenciar toda a força de trabalho. Os níveis de desemprego, por exemplo, são fortemente determinados por mudanças de período e não de coorte (Barros, Camargo \& Mendonça, 1997). O nível de renda, também é fortemente afetado por efeitos de período. No Brasil, as possibilidades de mobilidade social intergeracional também são afetadas por mudanças de período e não de coorte (Torche \& Ribeiro, 2010).

Tanto efeitos de coorte como de período estão relacionados a mudanças históricas. A diferença é que o efeito de coorte de idade está relacionado a algum momento do ciclo de vida, enquanto o efeito de período diz respeito a alguma mudança que afeta toda a população, independentemente do momento em que nasceram. Como destaquei acima, há ainda um terceiro efeito relacionado à idade das pessoas, que
1. Para estudos recentes sobre o Brasil, ver Collares (2010), Guimarães e Rios-Neto (2011), e Barbosa (2016) 
não é propriamente um efeito histórico, mas está diretamente relacionado ao ciclo de vida. O objetivo deste artigo é descrever as tendências históricas da inserção no mercado de trabalho dos indivíduos com educação superior no Brasil, mas, antes, apresento também as tendências de acesso à universidade. Comparo as tendências para homens e mulheres, e também descrevo as desigualdades de gênero.

\section{Dados e metodologia}

Metodologicamente é muito difícil distinguir os três efeitos porque há uma dependência linear exata: período $=$ coorte + idade. Este problema faz com que modelos para coorte, período e idade não possam ser matematicamente identificados. Uma longa tradição de estudos em sociologia, demografia e epidemiologia procurou soluções para este problema de identificação (Fienberg \& Mason, 1985; Mason, Mason, Winsborough \& Poole, 1973; O’Brien, 2000). Embora não haja uma solução inequívoca, dispomos de uma metodologia recentemente proposta que vem se mostrando bastante útil em diversos estudos (Yang, Fu \& Land, 2004; Yang, Schulhofer-Wohl, Fu \& Land, 2008). É justamente esta metodologia que utilizo neste trabaIho para analisar as tendências históricas de acesso à universidade e de inserção no mercado de trabalho de pessoas com educação superior.

Utilizo dados das Pesquisas Nacionais por Amostra de Domicílios (Pnad), que nos permitem construir uma série temporal de 25 anos de 1981 a 2006. Ao analisar os dados para pessoas entre 25 e 64 anos de idade em cada ano da Pnad, temos acesso às coortes de pessoas nascidas entre 1918 e 1981. Assim sendo, os dados permitem analisar tendências populacionais ocorridas entre 1918 e 2006 caso haja efeitos de coorte, ou mudanças entre 1981 e 2006 caso haja efeitos de período. De fato, os dados permitem traçar um panorama muito interessante sobre as possíveis relações entre as mudanças históricas que ocorreram no Brasil e as condições de trabalho das pessoas com escolaridade superior.

Os dados das Pnad foram organizados em tabelas com grupos de dois anos de período, de coorte e de idade. Desta forma, é possível estimar modelos para obter as taxas ponderadas por idade, período e coorte. Neste artigo analiso as seguintes taxas: (1) percentual da população que chegou ao ensino superior, e (2) percentual de pessoas com ensino superior que chegou ao topo da hierarquia ocupacional (profissionais e administradores, que correspondem à classe I do esquema usado por Ribeiro (2007). Apresento análises separadas para homens e mulheres.

A análise destes dados permite verificar em detalhe o acesso à educação superior e o destino ocupacional dos indivíduos com educação superior no Brasil em um período de grandes mudanças socioeconômicas e institucionais. Além do período de 
1981 a 2006, também analiso as coortes de nascimento (as assim chamadas coortes fictícias). Tendo em vista que as coortes incluem pessoas que nasceram entre 1918 e 1981, caso haja efeito de coorte confirmado pelos modelos estimados há a possibilidade de observarmos mudanças e tendências para um período mais longo de tempo, ou seja, efeitos nas perspectivas de alcançar a educação superior e uma posição ocupacional elevada no mercado de trabalho para pessoas que iniciaram e desenvolveram suas vidas educacional e laboral desde o final da década de 1930 até a década de 2000 .

\section{Resultados}

Nesta seção aponto os resultados encontrados em três blocos: (1) mostro as tendências para os homens; (2) descrevo as tendências para as mulheres; e (3) comparo as diferenças ou desigualdades nas tendências entre homens e mulheres.

\section{Homens}

Apresento a resposta aos modelos usados para explicar as tendências de acesso à universidade para toda a população de homens, bem como as oportunidades daqueles que concluíram a educação de terceiro grau se tornarem profissionais ou administradores, ou seja, chegarem ao topo da hierarquia ocupacional. Para analisar essas tendências históricas, estimei modelos levando em conta os efeitos dos grupos de idade de dois anos cada, entre 25 a 64 anos, das coortes de dois anos nascidas entre 1918 e 1981, e dos períodos de dois anos cada entre 1981 e 2006.

O modelo IPC estimado para explicar as tendências de acesso à universidade da população masculina no Brasil revela que há efeitos claros de período e coorte, e menos acentuados de idade. Em outras palavras, quando controlamos por período e coorte o efeito da idade diminui, mas o inverso não é verdade para o efeito do período controlando por idade e coorte, ou da coorte controlando por idade e período.

O Gráfico 1 indica que as pessoas entre 29 e 41 anos têm mais acesso à universidade do que os mais novos e os mais velhos. Ao controlar pelo efeito de coorte e período (I/PC) verifiquei que a diferença diminui. Embora haja uma distância entre as taxas líquida e bruta, o modelo estimado revela que a tendência ao longo dos grupos de idade é estatisticamente significativa, o que me leva a concluir que a taxa líquida estimada corrige o que se observa nas taxas brutas. Ou seja, o efeito da idade sobre a proporção com acesso à universidade não é tão acentuado quanto o observado nas taxas brutas. De fato, ao corrigir a tendência verifiquei que até os 29-30 anos de idade há um aumento da participação na universidade, mas depois desta idade há uma diminuição monotônica da participação. 
GRÁFICO 1

PROPORÇÃO DE HOMENS COM ALGUMA EDUCAÇÃO

DE NÍVEL UNIVERSITÁRIO NO BRASIL POR GRUPO DE IDADE:

TAXAS BRUTAS E TAXAS CONTROLANDO POR PERÍODO E COORTE (I/PC)

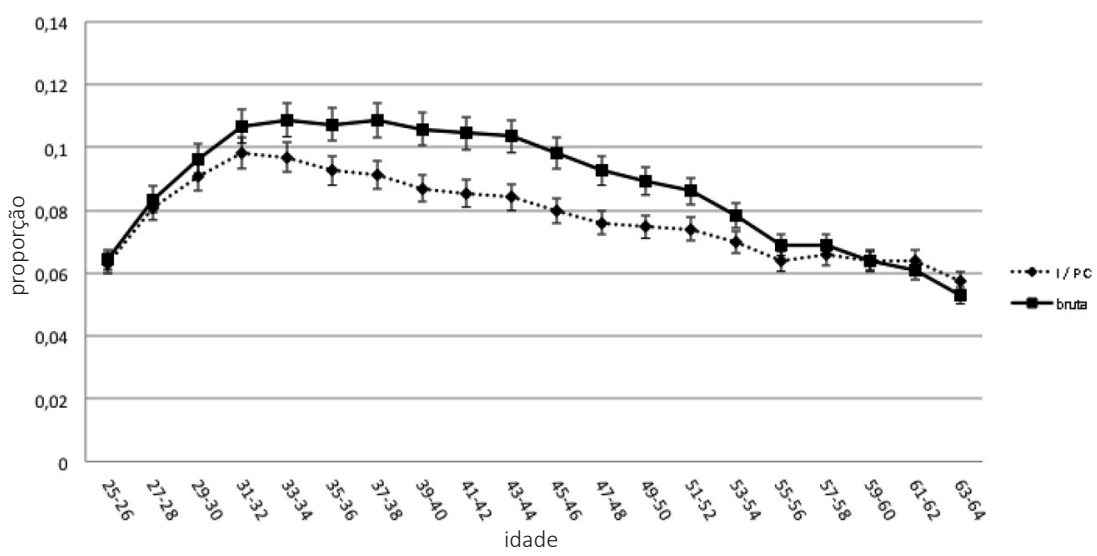

GRÁFICO 2

PROPORÇÃO DE HOMENS COM ALGUMA EDUCAÇÃO

DE NÍVEL UNIVERSITÁRIO NO BRASIL POR GRUPO DE PERÍODO (1981 A 2006):

TAXAS BRUTAS E TAXAS CONTROLANDO POR IDADE E COORTE (P/IC)

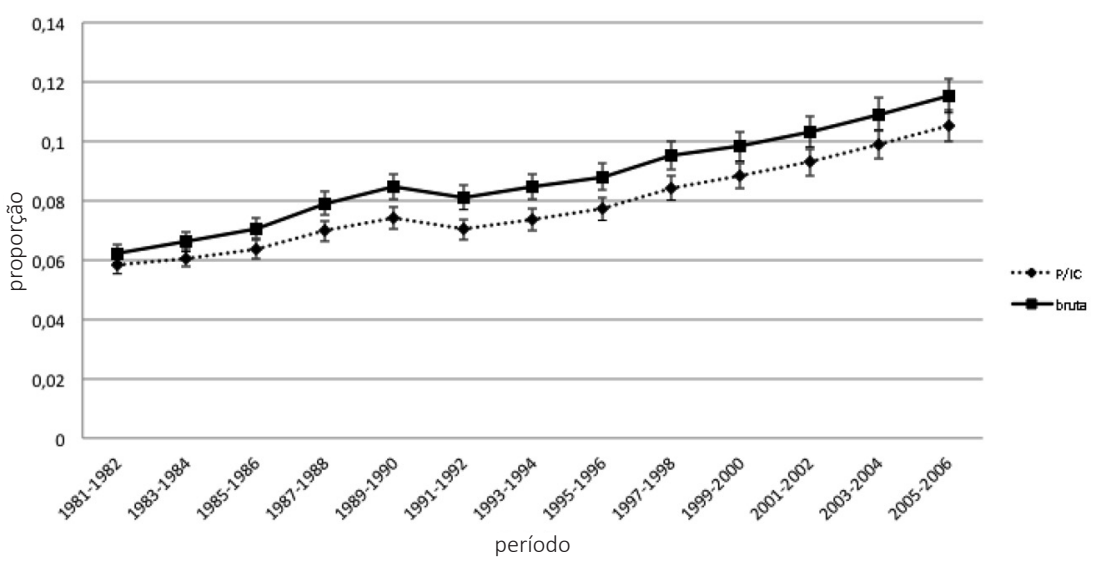

O Gráfico 2 apresenta as tendências ao longo do período que vai de 1981 a 2006. Observa-se uma clara tendência de aumento do acesso à universidade ao longo das décadas no Brasil. As taxas brutas não são muito diferentes das taxas líquidas, o que significa que o efeito do período é forte e estatisticamente significativo.

Finalmente, é possível observar, no Gráfico 3, uma clara tendência de aumento do acesso à universidade para as coortes nascidas entre 1918 e 1948. Estes homens 
GRÁFICO 3

PROPORÇÃO DE HOMENS COM ALGUMA EDUCAÇÃO

DE NÍVEL UNIVERSITÁRIO NO BRASIL POR GRUPO COORTE DE NASCIMENTO:

TAXAS BRUTAS E TAXAS CONTROLANDO POR PERÍODO E IDADE (C/IP)

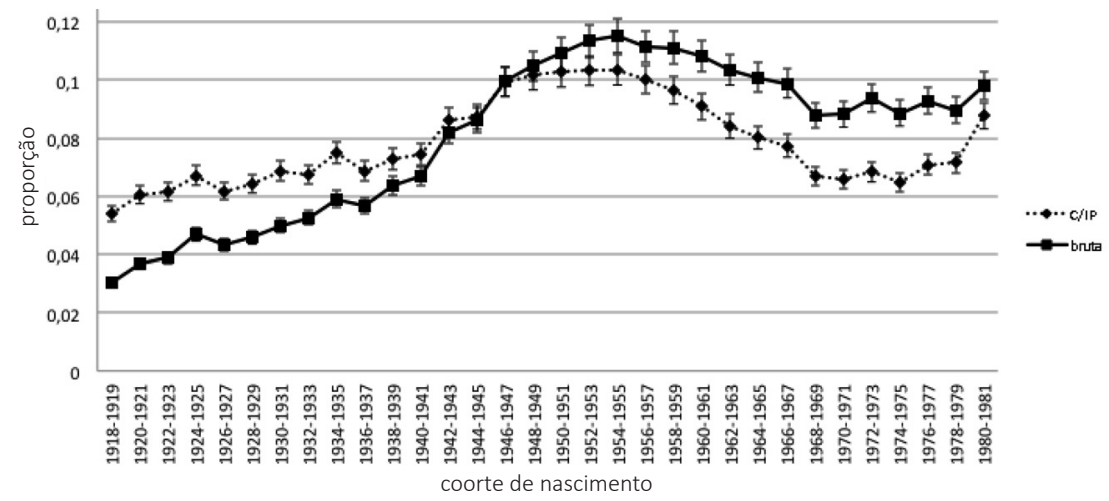

tinham 20 anos de idade entre 1938 e 1968 e, portanto, estavam na idade típica de frequentar a universidade neste período. Reparem que este é um período de expansão econômica. Em contraste, as coortes nascidas entre 1956 e 1968, que tinham 20 anos entre 1976 e 1988, apresentam uma diminuição do acesso à universidade. Ora, estas coortes são justamente aquelas que deveriam estar na universidade durante o período em que a economia brasileira passou por uma de suas crises mais marcantes. A partir de 1976 - o período do chamado "Milagre econômico" -, começou a entrar em colapso e na década de 1980, depois da crise internacional de crédito, que solapou toda América Latina, o país praticamente não cresceu. As coortes que viveram este período, no momento em que poderiam estar na universidade, sofreram claramente os efeitos da crise. Estes efeitos tendem a durar por toda a vida destes homens, tendo em vista que com o envelhecimento as possibilidades de entrar na universidade diminuem consideravelmente, como vimos no Gráfico 1.

As análises resumidas nos Gráficos 1, 2 e 3 revelam, por um lado, que há uma tendência de aumento do acesso à universidade entre 1981 e 2006, e, por outro, que há um aumento seguido de queda do acesso à universidade ao longo das coortes de nascimento. Esta última tendência indica que as oportunidades de acesso à universidade no Brasil são concomitantes às flutuações econômicas de crescimento (até o final da década de 1970) e à crise (a partir da década de 1980). Mas será que os indivíduos que chegaram à universidade tiveram sua situação no mercado de trabalho afetada? 


\section{GRÁFICO 4}

PROPORÇÃO DE HOMENS COM EDUCAÇÃO

DE NÍVEL UNIVERSITÁRIO NO TOPO DA HIERARQUIA OCUPACIONAL NO BRASIL POR GRUPO DE IDADE: TAXAS BRUTAS E TAXAS CONTROLANDO POR PERÍODO E COORTE (I/PC)

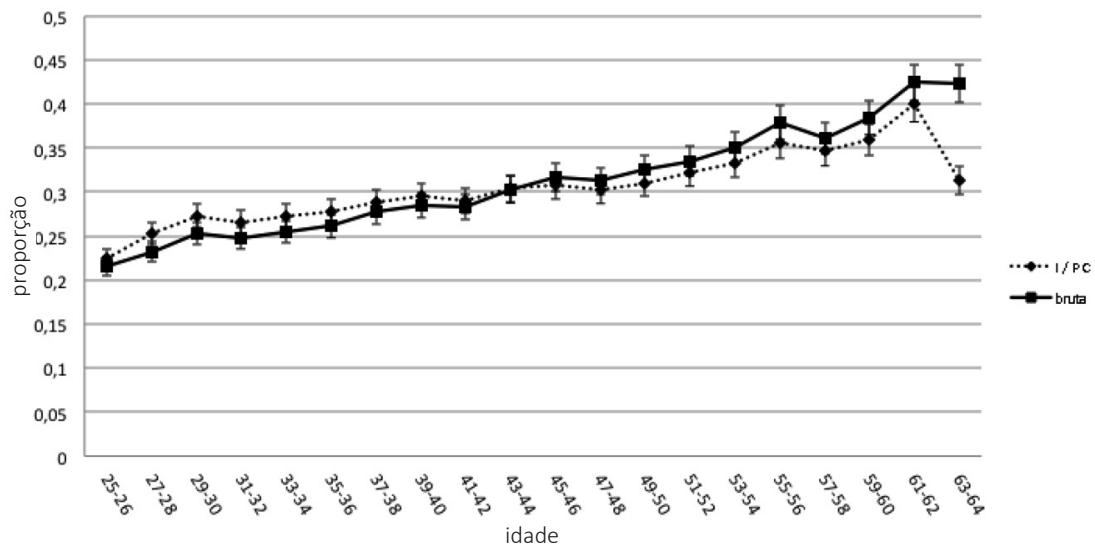

Para responder a essa pergunta, estimei um modelo de idade, período e coorte para analisar as possibilidades de os indivíduos com educação de nível superior ocuparem posições de profissionais ou administradores, ou seja, ocupações no topo da hierarquia socioeconômica. Os resultados desse modelo indicam que as coortes de nascimento não explicam bem a tendência de acesso a tais ocupações de elite. O Gráfico 5 mostra que há uma tendência ao longo do período (não das coortes) de diminuição da proporção de pessoas com educação universitária ocupando posições de profissionais e administradores entre 1981 e 2006. Em contrapartida, quando controlando pelo efeito do período e da idade, a tendência de diminuição ao longo das coortes (Gráfico 6) praticamente desaparece. Em outras palavras, o modelo confirma as tendências ao longo dos grupos de idade (aumento da proporção com a idade) e ao longo dos períodos observados (diminuição da proporção ao longo do tempo), e corrige a tendência ao longo das coortes. Conclui-se, por um lado, que há diminuição do acesso ao topo da hierarquia ocupacional, entre 1981 e 2006, e aumento de acesso a estas ocupações com a idade e, por outro lado, que não há tendência para as coortes de idade.

Substantivamente, isso significa que toda a força de trabalho com ensino superior (independentemente da coorte de nascimento) está cada vez menos alcançando posições no topo da hierarquia ocupacional. Este efeito é ainda maior para os mais jovens. Ou, inversamente, cada vez mais as pessoas com educação superior estão em posições de menor status socioeconômico na hierarquia ocupacional. De fato, estes resultados confirmam os anteriormente encontrados em outros estudos que mostram haver uma diminuição dos retornos educacionais (ou uma inflação de 


\section{GRÁFICO 5}

PROPORÇÃO DE HOMENS COM EDUCAÇÃO

DE NÍVEL UNIVERSITÁRIO NO TOPO DA HIERARQUIA OCUPACIONAL NO BRASIL POR PERÍODO: TAXAS BRUTAS E TAXAS CONTROLANDO POR IDADE E COORTE (P/IC)



GRÁFICO 6

PROPORÇÃO DE HOMENS COM EDUCAÇÃO

DE NÍVEL UNIVERSITÁRIO NO TOPO DA HIERARQUIA OCUPACIONAL NO BRASIL

POR COORTE DE IDADE: TAXAS BRUTAS E TAXAS CONTROLANDO POR IDADE E PERÍODO (C/IP)

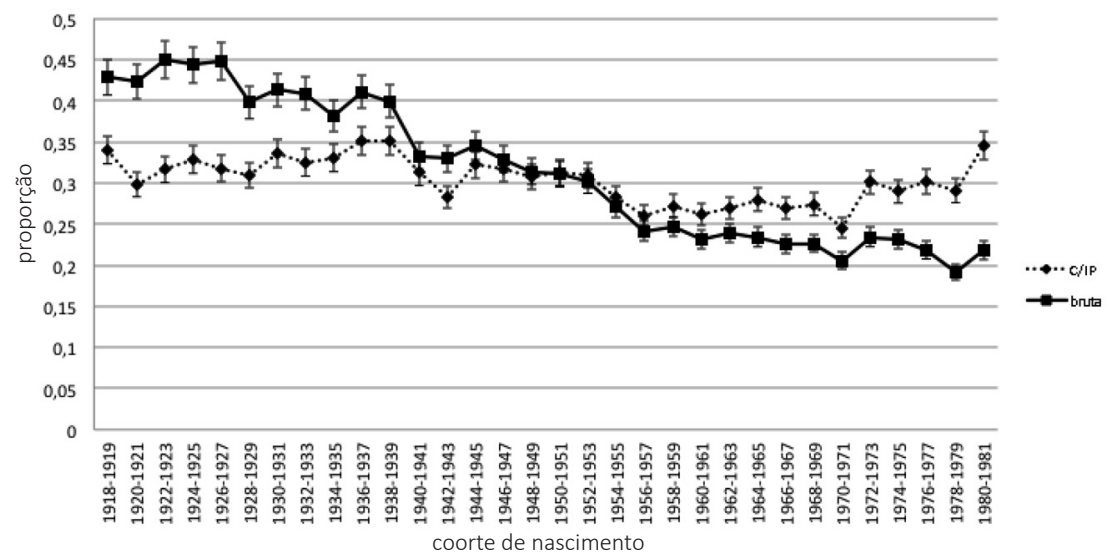

credenciais) no Brasil (Ferreira et alii, 2006; Torche \& Ribeiro, 2010). Isso significa que a relação entre educação de ensino superior e ocupações está mudando significativamente no Brasil durante as últimas três décadas. O que era uma ocupação típica de pessoas com ensino superior em 1981 não o é mais no final da década de 2000. 


\section{Mulheres}

A situação educacional e o mercado de trabalho das mulheres são bastante distintos em relação aos dos homens. Diversos estudos mostram que há um aumento muito grande, ao longo dos anos, do acesso das mulheres ao sistema educacional, incluindo à universidade (Barros et alii, 2001). Na realidade, o desempenho educacional das mulheres ultrapassou mesmo o dos homens, tendo em vista que, em média, elas permanecem mais tempo na escola do que eles. No mercado de trabalho, no entanto, os homens estão em posições hierarquicamente superiores, têm salários mais elevados e participação maior em termos percentuais. Embora as mulheres estejam crescentemente entrando no mercado de trabalho, os homens continuam em situação de vantagem. Por estas razões o acesso das mulheres ao ensino superior e a colocação daquelas com educação superior no mercado de trabalho guarda especificidades e deve ser observado separadamente.

Embora as características educacionais e do mercado de trabalho das mulheres sejam distintas das dos homens, os modelos estimados para descrever os efeitos de idade, coorte e período usando os dados para mulheres revelam tendências semeIhantes àquelas observadas para os homens. O modelo para o acesso à universidade indica que o efeito de idade é muito parecido ao obtido para os homens, ou seja, aumento da proporção até 33 ou 34 anos e diminuição após esta idade (Gráfico 7). O efeito mais claro, no entanto, é o de período. Há um aumento do acesso à universidade ao longo dos anos entre 1981 e 2006. Tendo em vista que esse aumento se

\section{GRÁFICO 7}

PROPORÇÃO DE MULHERES COM ALGUMA EDUCAÇÃO DE NÍVEL UNIVERSITÁRIO NO BRASIL POR GRUPO DE IDADE:

TAXAS BRUTAS E TAXAS CONTROLANDO POR PERÍODO E COORTE (I/PC)

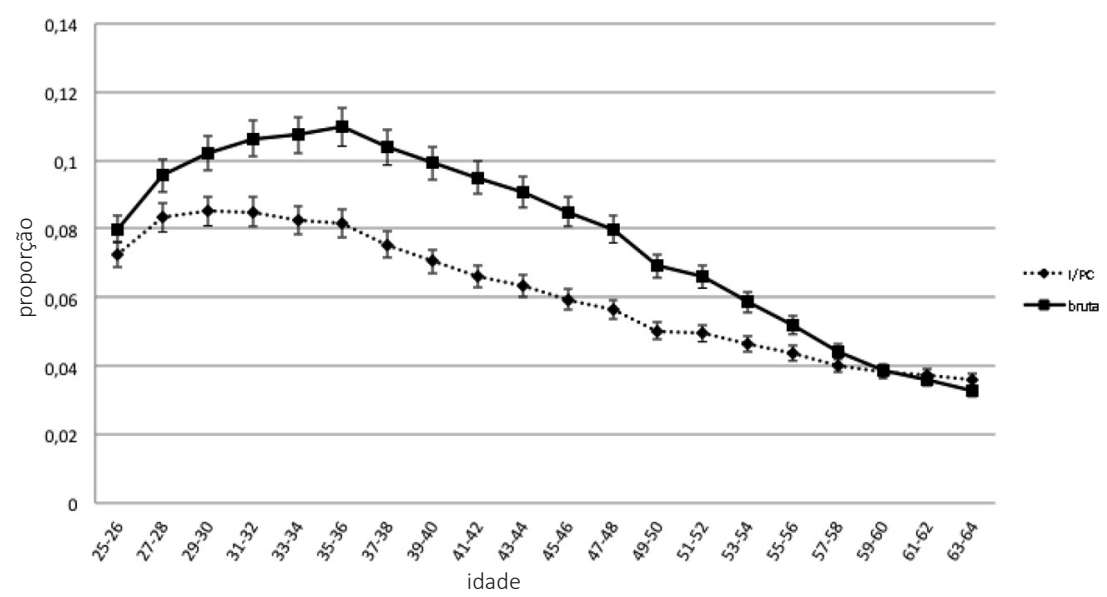


GRÁFICO 8

PROPORÇÃO DE MULHERES COM ALGUMA EDUCAÇÃO

DE NÍVEL UNIVERSITÁRIO NO BRASIL POR GRUPO PERÍODO (1981-2006):

TAXAS BRUTAS E TAXAS CONTROLANDO POR IDADE E COORTE (P/IC)

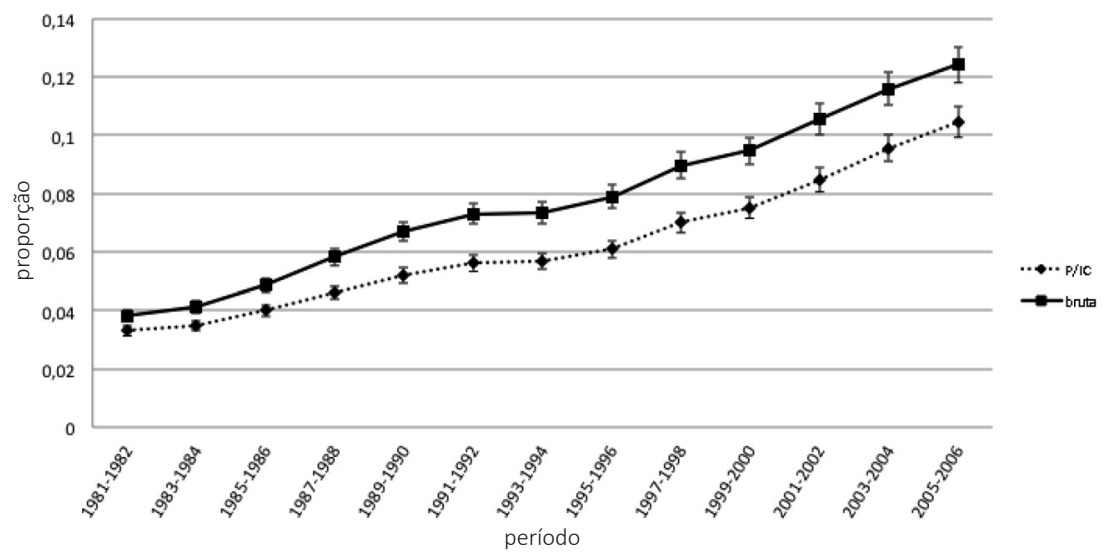

GRÁFICO 9

PROPORÇÃO DE MULHERES COM ALGUMA EDUCAÇÃO

DE NÍVEL UNIVERSITÁRIO NO BRASIL POR COORTE DE NASCIMENTO:

TAXAS BRUTAS E TAXAS CONTROLANDO POR PERÍODO E IDADE (C/IP)

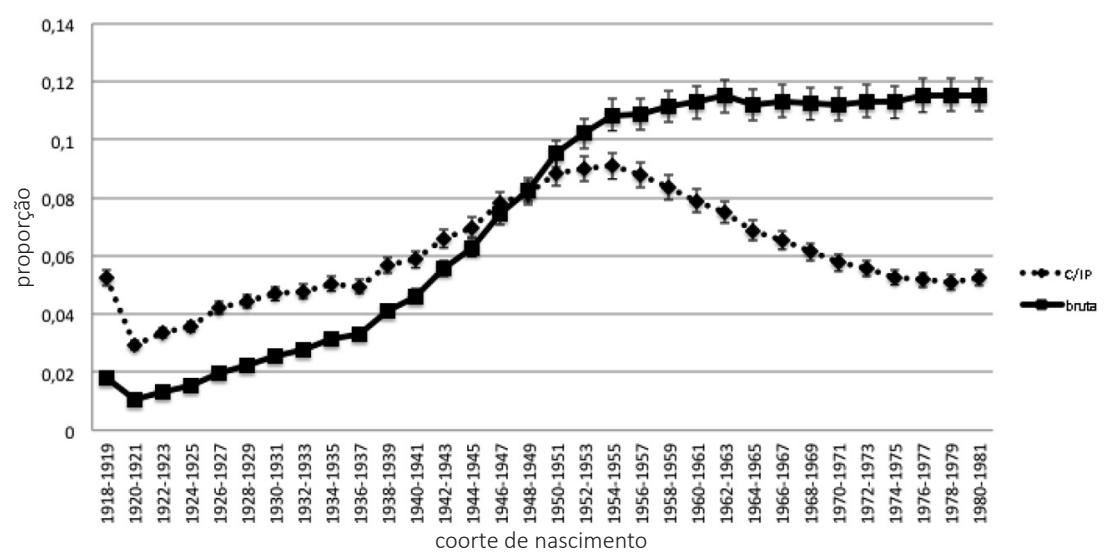

dá mesmo quando levamos em conta idade e coorte, devemos concluir que é um aumento afetando todas as mulheres.

Uma conclusão completamente diferente surge quando comparamos a tendência observada para as coortes de nascimento com a estimada (controlando pelos efeitos de período e idade). No Gráfico 9, as tendências brutas indicam que há um aumento na proporção de mulheres entrando na universidade até as coortes nascidas 
entre 1958 e 1961, e depois a proporção permanece estável. As tendências estimadas (ou ponderadas) são completamente diferentes: mostram uma diminuição do acesso das mulheres à universidade para as coortes nascidas a partir de $1956 \mathrm{e}$ 1957. As mulheres nestas coortes mais jovens tinham 20 anos no final da década de 1970 e durante a década de 1980, período em que o país passou por séria crise econômica. Assim como ocorre para as coortes de homens, há uma diminuição do acesso à universidade durante a década de 1980 para as mulheres, ou seja, as condições macroeconômicas de crise são concomitantes com a uma diminuição do acesso da população à universidade. Isso ocorreu provavelmente porque as pessoas tiveram de optar por trabalhar ao invés de se dedicar aos estudos. É importante destacar que um pesquisador olhando apenas para as taxas brutas das coortes fictícias chegaria a conclusões erradas sobre as tendências de acesso à universidade das mulheres nascidas em diferentes momentos.

Também estimei um modelo para descrever as taxas ponderadas de mulheres com educação superior que chegaram a ocupações no topo da hierarquia (profissionais e administradores). Os Gráficos 10, 11 e 12 apresentam os resultados destes modelos e revelam que as taxas brutas e as estimadas estão muito próximas, o que implica dizer que a observação das taxas brutas não leva a conclusões erradas, como seria o caso do acesso à universidade apresentado acima.

Curiosamente as análises indicam que mulheres mais velhas nascidas em coortes mais antigas (Gráfico 10 e 12) com educação universitária têm maiores possibilida-

GRÁFICO 10

PROPORÇÃO DE MULHERES COM EDUCAÇÃO DE NÍVEL UNIVERSITÁRIO NO TOPO DA HIERARQUIA OCUPACIONAL NO BRASIL POR GRUPO DE IDADE: TAXAS BRUTAS E TAXAS CONTROLANDO POR PERÍODO E COORTE (C/IP)

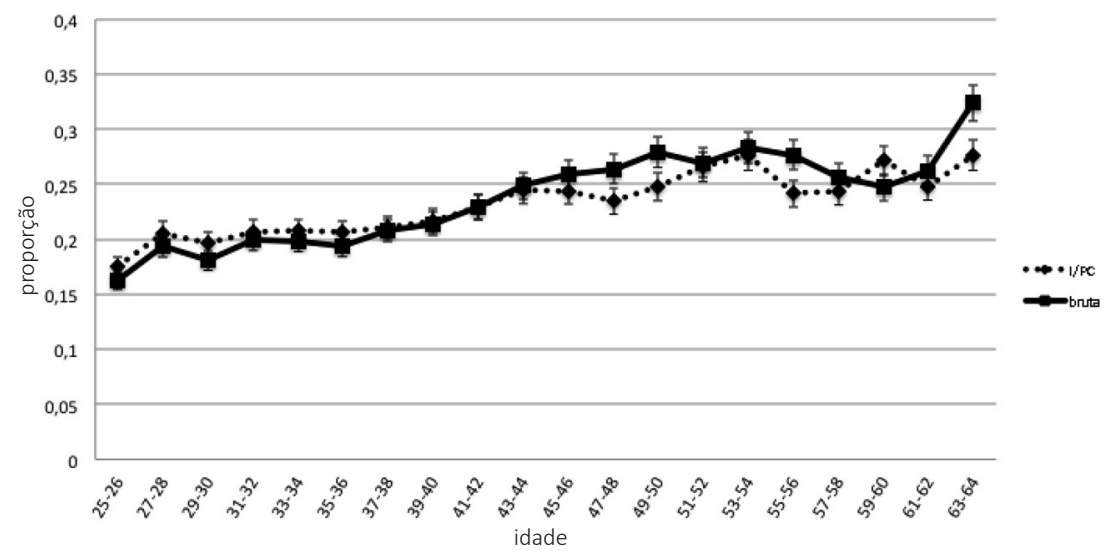


GRÁFICO 11

PROPORÇÃO DE MULHERES COM EDUCAÇÃO DE NÍVEL UNIVERSITÁRIO NO TOPO DA HIERARQUIA OCUPACIONAL NO BRASIL POR PERÍODO: TAXAS BRUTAS E TAXAS CONTROLANDO POR IDADE E COORTE (P/IC)

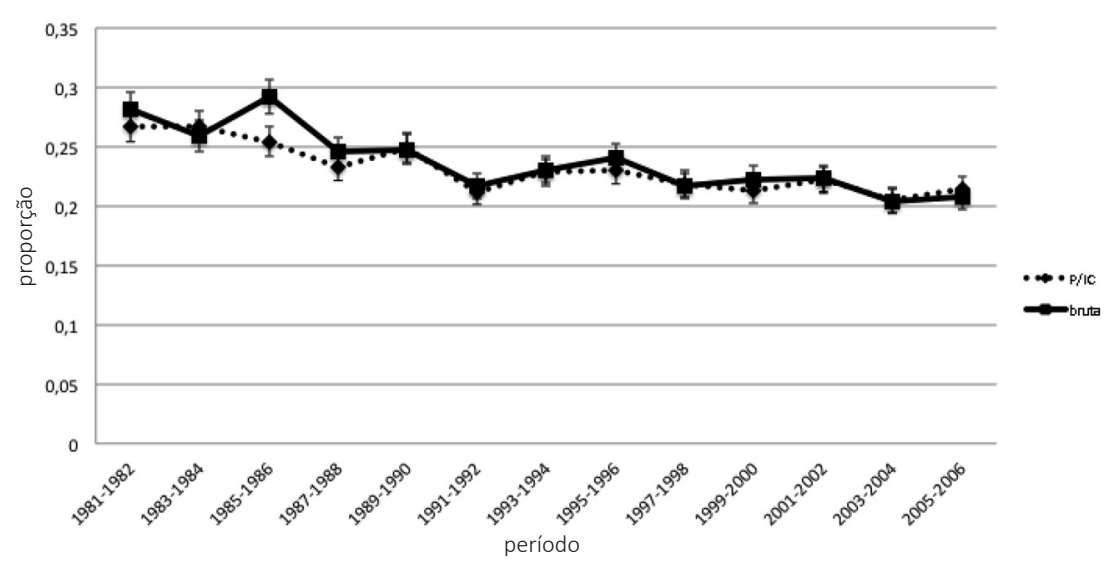

GRÁFICO 12

PROPORÇÃO DE MULHERES COM EDUCAÇÃO DE NÍVEL UNIVERSITÁRIO NO TOPO DA HIERARQUIA OCUPACIONAL NO BRASIL POR COORTE DE IDADE: TAXAS BRUTAS E TAXAS CONTROLANDO POR IDADE E PERÍODO (C/IP)



des de ocupar posições de profissionais e administradoras do que as mais novas. Este resultado se complementa por um forte efeito de diminuição do acesso de mulheres com educação superior às posições no topo da hierarquia ocupacional ao longo do período que vai de 1981 e 2006. De fato, as tendências para idade e coorte estão fortemente influenciadas pela tendência ao longo do período. Enquanto em 1981 cerca de $30 \%$ das mulheres com educação superior estavam em posições de 
profissionais e administradoras, em 2006 apenas 20\% estavam em tais posições. Sendo assim, não é possível dizer que as ocupações típicas de mulheres com educação universitária em 1981 fossem as mesmas em 2006. Outra maneira de entender esta tendência é dizer que está havendo uma "inflação de credenciais" ou que os diplomas universitários estão perdendo o seu valor. É mais comum observar muIheres com diplomas universitários em ocupações hierarquicamente inferiores em 2006 do que em 1981.

\section{Desigualdade de gênero}

As análises apresentadas acima para mulheres e para homens não entabulam uma comparação direta entre os dois grupos. Neste sentido, os gráficos apresentados não permitem dizer se há desigualdade de gênero no acesso à universidade e às ocupações no topo da hierarquia. Nesta seção descrevo as tendências da desigualdade de gênero no acesso ao topo da hierarquia ocupacional, mas não apresento a análise para acesso à universidade. Para fazer uma análise correta do acesso à universidade eu deveria verificar o percentual das pessoas com ensino médio completo que chegou a universidade e não o percentual de toda a população que chegou a universidade. Esta segunda abordagem levaria a resultados errôneos na medida em que há diferença significativa nos percentuais de homens e mulheres completando o ensino médio, ou seja, há desigualdade de gênero na progressão educacional anterior à entrada no ensino superior. Isto significa que se compararmos a proporção de toda a população de homens e de mulheres que chegou à universidade maximizaríamos a desigualdade observada.

Este problema não se coloca para a análise do acesso ao topo da hierarquia ocupacional, tendo em vista que esta análise tem como base apenas as pessoas com nível superior de ensino.

Tendo em vista que tanto para homens como para mulheres observamos uma forte tendência de diminuição do acesso ao topo da hierarquia ocupacional ao longo do período de 1981 e 2006, é importante verificarmos se houve alguma tendência na desigualdade de gênero neste acesso. De fato, ao compararmos os percentuais observamos que a tendência de diminuição do acesso dos homens a posições no topo da hierarquia ocupacional foi maior do que a tendência de diminuição para as mulheres. Entre 1981 e 2006, houve uma diminuição de 10,9\% para os homens e de apenas 5,3\% para as mulheres. Podemos tirar duas conclusões destes dados. Por um lado, podemos dizer que houve uma diminuição na desigualdade de gênero entre homens e mulheres com educação universitária entre 1981 e 2006. Por outro, podemos levantar a hipótese de que parte da diminuição do acesso ao topo da hierarquia ocupacional entre os homens com ensino superior se deve à competição com as mulheres com qualificações semelhantes. Em suma, a desigualdade de gê- 
nero diminuiu em parte porque as mulheres estão competindo com os homens pelas melhores ocupações. Tendo em vista que no período estudado realmente houve uma expansão da participação feminina no mercado de trabalho, podemos concluir que a crescente dificuldade de acesso ao topo da hierarquia ocupacional que os homens enfrentaram se deve justamente ao aumento da participação feminina e da competição entre homens e mulheres com educação universitária.

\section{Conclusões}

As análises apresentadas permitem chegar a diversas conclusões importantes sobre as tendências históricas de acesso à universidade e às ocupações no topo da hierarquia para a população brasileira. As análises permitem observar tendências históricas relacionadas a efeitos de coorte de nascimento para acesso à universidade e a efeitos de período para acesso às ocupações no topo da hierarquia. Os efeitos de coorte mostram que, durante a crise econômica das décadas de 1980 e 1990, diminuíram as possibilidades de acesso à universidade para os indivíduos que chegaram à idade típica de estar no ensino superior nestas décadas. Embora não seja possível dizer que a crise econômica causou a diminuição do acesso à universidade, os dados analisados indicam que esta hipótese deve ser investigada com mais cuidado. Os efeitos de período, por sua vez, indicam que, entre 1981 e 2006, houve um processo de "inflação de credenciais" diminuindo as perspectivas de os indivíduos com educação universitária chegarem ao topo da hierarquia ocupacional. Na medida em que a ocupação é um indicador de renda permanente, este último processo está relacionado à diminuição do "prêmio salarial ou de renda" conferido pelo diploma universitário, que é um dos fatores que contribui para a diminuição da desigualdade de renda no Brasil (Ferreira et alii, 2006).

Para a população masculina com idade entre 25 e 64 anos, observamos uma tendência de aumento do acesso à universidade ao longo do período que vai de 1981 a 2006. Este aumento no percentual de pessoas com educação universitária pode ter contribuído para um processo de "inflação de credenciais". De fato, uma maior oferta de indivíduos com educação universitária que não seja acompanhada de uma maior demanda por essa mão de obra pode levar a um processo de desvalorização dos diplomas universitários. Tendo em vista que o percentual de pessoas com ensino universitário alcançando posições de profissionais e administradores também diminuiu ao longo dos anos ou do período (como observamos no Gráfico 5), podemos concluir que há uma diminuição na demanda por pessoas com nível universitário. Portanto, deve realmente estar ocorrendo um processo de "inflação de credenciais".

Paralelamente, houve uma queda do percentual de homens com acesso à universidade a partir da coorte nascida entre 1956 e 1968. Estas coortes estavam na idade 
típica de frequentar a universidade (20 anos) durante a década de 1980. Portanto, também podemos concluir que suas chances educacionais estão relacionadas de alguma forma à crise econômica dos anos 1980. O acesso dos homens com educação universitária às ocupações no topo da hierarquia, no entanto, não muda significativamente ao longo das coortes de idade (Gráfico 6), ou seja, não há efeito de coorte, mas apenas de período (Gráfico 5). Isto significa que a diminuição do acesso a posições no topo da hierarquia ocupacional afetou toda a população com ensino superior, independentemente do momento em que nasceram. No período de 1981 a 2006, houve uma enorme desvalorização dos diplomas de ensino superior no mercado de trabalho. Neste sentido, ocupações típicas de pessoas com ensino superior em 1981 não são as mesmas em 2006.

As tendências encontradas para as mulheres são muito semelhantes àquelas descritas para os homens. No entanto, a comparação das tendências observadas para homens e mulheres mostra que há uma diminuição da desigualdade de gênero entre 1981 e 2006. De fato, esta conclusão é importante porque indica que parte da diminuição do acesso dos homens às posições de profissionais e administradores se deve ao acesso das mulheres a esses tipos de ocupação. Podemos mesmo levantar a hipótese de que a desvalorização dos diplomas universitários ("inflação de credenciais"), ao longo do período estudado, se deve em parte à crescente participação feminina na força de trabalho com qualificação universitária.

A diminuição do acesso ao topo da hierarquia ocupacional observada nas análises afeta toda a população e não apenas algumas coortes. Isso significa que toda a população com educação universitária sofreu uma diminuição de suas oportunidades de acesso às ocupações de profissionais e administradores. Provavelmente, diversas pessoas perderam posições no topo da hierarquia ocupacional ao longo do período estudado, ou seja, experimentaram mobilidade ocupacional descendente. As comparações entre homens e mulheres indicam que parte desta diminuição no percentual com acesso ao topo da hierarquia ocupacional se deve à crescente participação das mulheres no mercado de trabalho para pessoas com ensino universitário. Mas provavelmente há outros fatores em jogo, como, por exemplo: (1) diversificação da qualidade dos diplomas universitários; (2) aumento de exigências por educação superior para ocupações inferiores na escala ocupacional; (3) descompasso entre

2. Para uma análise mais sofisticada nesta linha, veja Barbosa (2016). a criação de posições ocupacionais no topo da hierarquia e oferta de pessoas com educação superior, entre outros. Espero que as análises que desenvolvi sobre idade, período e coorte sejam mais usadas e estudadas nas ciências sociais brasileiras². 
Abstract: This paper studies trends in men's and women's entry into college and into the labor market for those with college diplomas between 1981 and 2006 in Brazil. Taking into account that these individuals were born between 1918 and 1981, since they were between 25 and 64 years old when interviewed, the analysis are also about the effects of birth cohort that were born since 1918. In order to verify the effects of period, cohort and age I use models that allow to untangle this three temporal effects. The main objective, however, is to analyze if the cycles of development and economic expansion (until the end of the 1970's) and the period of stagnation and crisis during the 1980's and 1990's relate to populational trends of entry into university and into the labor market for those with university degrees. The paper shows evidence of cohort and period effect and also of decline in gender inequality.

Keywords: gender inequality, higher education, labor market.

\section{Referências}

BARBOSA, R. Desigualdade de rendimentos do trabalho no curto e no longo prazo: tendências de idade, período e coorte. Dados, Revista de Ciências Sociais, v. 59, n. 2, p. 33-71. Rio de Janeiro, 2016.

BARROS, R.; CORSEUIL, C.; SANTOS, D.; FIRPO, S. Inserção no mercado de trabaIho: diferenças por sexo e consequências sobre o bem-estar. Texto para discussão Ipea, n.796. Rio de Janeiro: Ipea, 2001.

BARROS, R. P.; CAMARGO, J.; MENDONÇA, R. A estrutura do desemprego no Brasil. Texto para discussão Ipea, n. 478. Rio de Janeiro: Ipea, 1997.

BREEN, R.; JONSSON, J. Inequality of opportunity in comparative perspective: recent research on educational attainment and social mobility. Annual Review of Sociology, v. 31, p. 223-243, 2005.

BREEN, R. E JONSSON, J. Explaining change in social fluidity: educational equalization and educational expansion in twentieth-century Sweden. American Journal of Sociology, 112 (6), p. 1775-1810, 2007.

COLLARES, A. C. Social inequality and the expansion of higher education in Brazil. (Ph.D. Dissertation) - Sociology Department, University of Wisconsin-Madison, 2010.

FERREIRA, F.; LEITE, P.; LITCHFIELD, J.; ULYSSEA, G. Ascensão e queda da desigualdade de renda no Brasil. Econômica, v. 8, n. 1, p. 147-169, 2006.

FIENBERG, S.; MASON, W. Specification and implementation of age, period and cohort models. In: MASON, W.; FIENBERG, E. (Orgs.). Cohort analysis in social research, p. 45-88. New York: Springer-Verlag, 1985. 
GUIMARÃES, R.; RIOS-NETO, E. Comparação entre metodologias de idade-período-coorte para o estudo de uma medida da progressão escolar no Brasil. Revista Brasileira de Estudos de População, v. 28, p. 349-367, 2011.

MASON, K.; MASON, W.; WINSBOROUGH, H.; POOLE, W. Some methodological issues in cohort analysis of archival data. American Sociological Review, v. 38, p. 242-258, 1973.

O'BRIEN, R. Age-period-cohort characteristic models. Social Science Research, v. 29, p. 123-139, 2000.

RIBEIRO, C. A. C. Desigualdade de oportunidades e resultados educacionais no Brasil. Dados, v. 54, n. 1, 2011.

Desigualdade de oportunidades educacionais no Brasil: raça, classe e gênero. In: RIBEIRO, C. Desigualdade de oportunidades no Brasil. Belo Horizonte: Argvmentvm, 2009.

SHAVIT, Y.; BLOSSFELD, H. P. Persistent inequality. Changing Educational Attainmet in Thirteen Countries. Boulder CO, 1993.

TORCHE, F.; RIBEIRO, C. C. Pathways of change in social mobility: industrialization, education and growing fluidity in Brazil. Research in Social Stratification and Mobility, v. 28, n. 3, p. 291-307, 2010.

YANG, Y.; FU, W. J.; LAND, K. A methodological comparison of age-period-cohort models: intrinsic estimator and conventional generalized linear models. Sociological Methodology, Sage Journals, 2004.

YANG, Y.; SCHULHOFER-WOHL, S.; FU, W. J.; LAND, K. The intrinsic estimator for age-period-cohort: what it is and how to use it? American Journal of Sociology, v. 113, n. 6, 2008. 


\section{Anexos}

\section{TABELA A1 \\ MODELOS IPC \\ (INTRINSIC ESTIMATOR)}

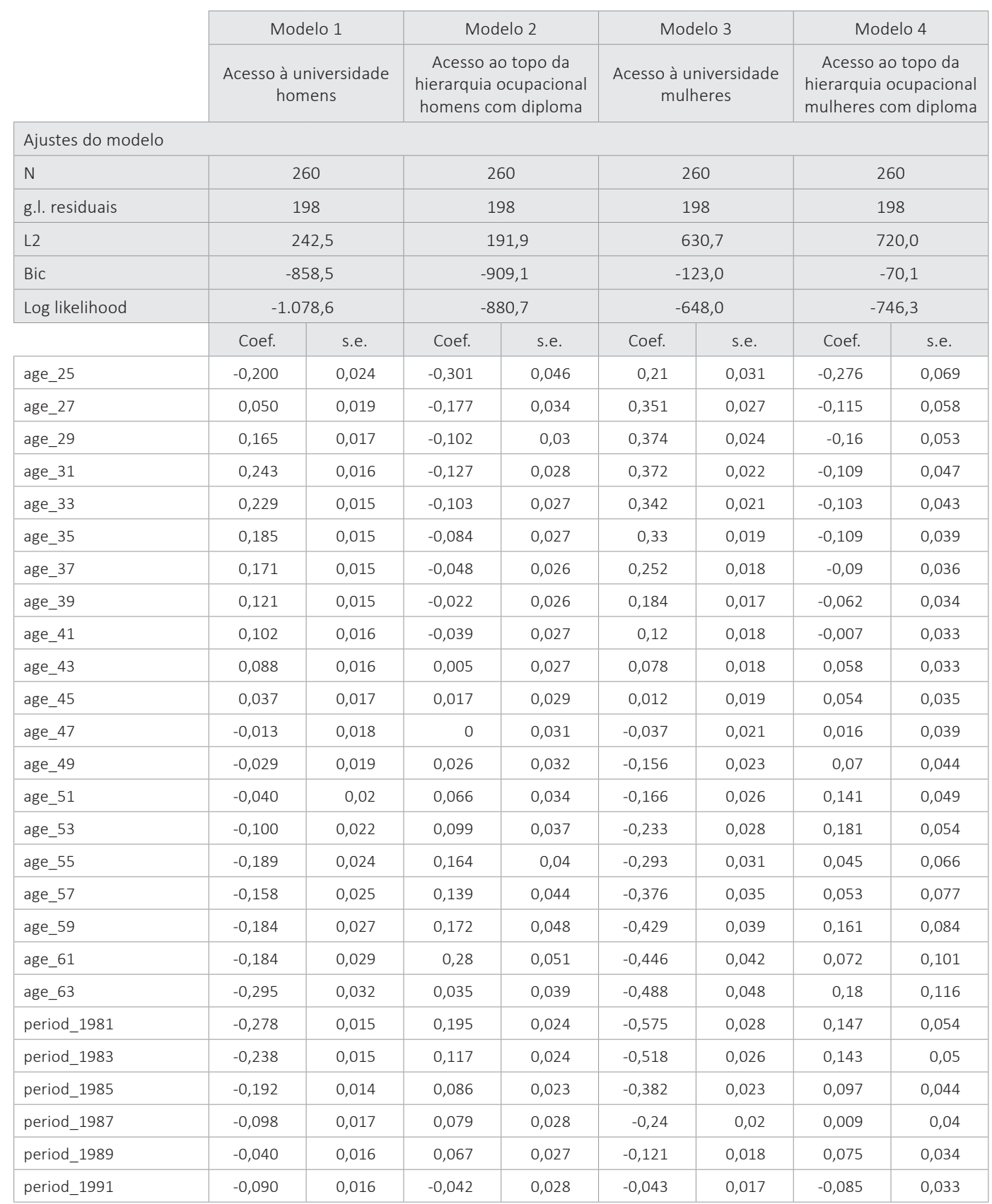









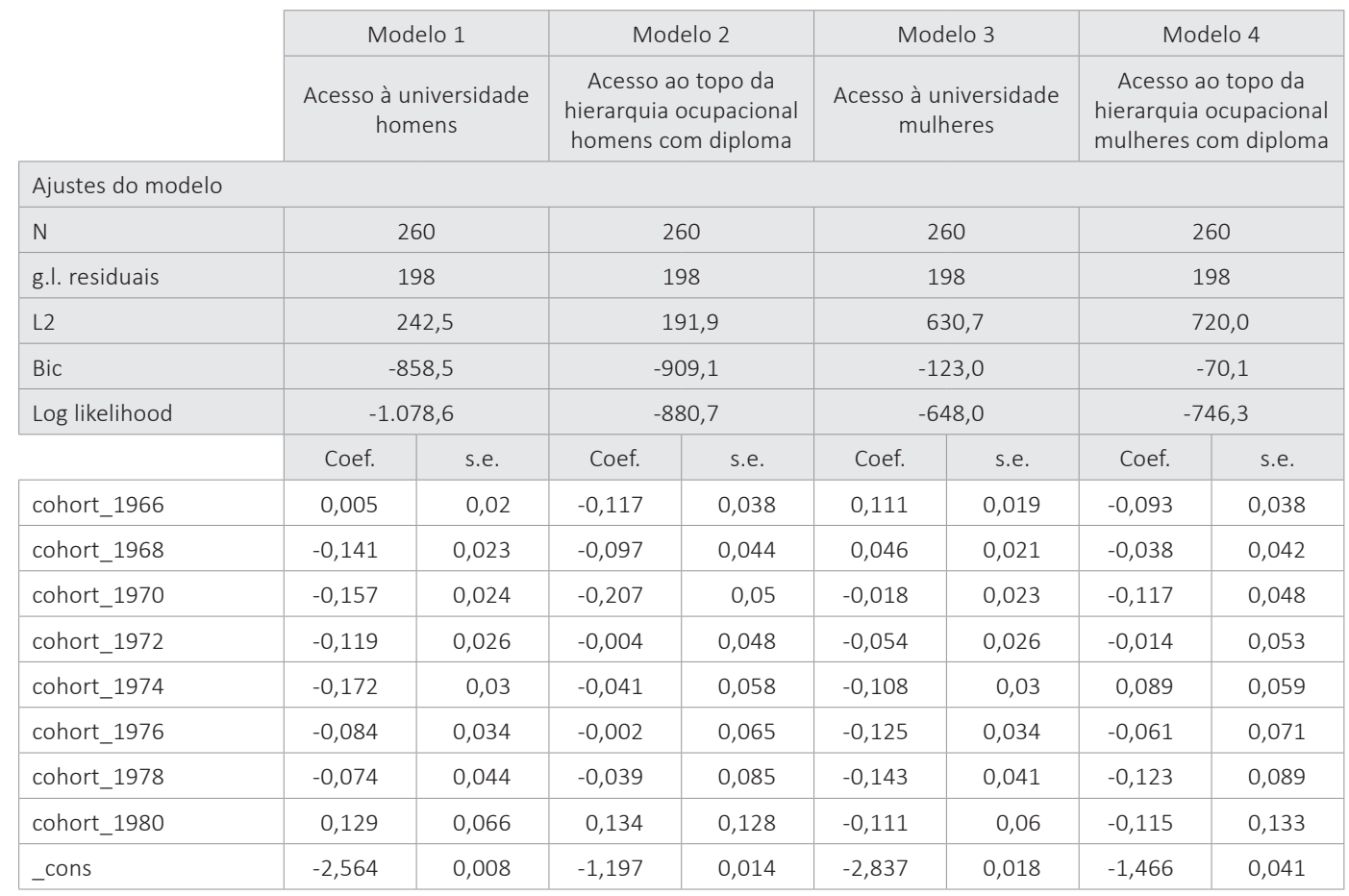


TABELA A2

MODELOS IPC

(INTRINSIC ESTIMATOR)

\begin{tabular}{|c|c|c|c|c|}
\hline & \multicolumn{2}{|c|}{ Modelo 1} & \multicolumn{2}{|c|}{ Modelo 2} \\
\hline & \multicolumn{2}{|c|}{$\begin{array}{l}\text { Acesso à universidade } \\
\text { brancos e não brancos }\end{array}$} & \multicolumn{2}{|c|}{$\begin{array}{c}\text { Acesso ao topo da hierarquia ocupaciona } \\
\text { brancos e não brancos com diploma } \\
\text { universitário }\end{array}$} \\
\hline \multicolumn{5}{|c|}{ Ajustes do modelo } \\
\hline N & \multicolumn{2}{|c|}{117} & \multicolumn{2}{|c|}{177} \\
\hline g.I. residuais & \multicolumn{2}{|c|}{77} & \multicolumn{2}{|c|}{77} \\
\hline L2 & \multicolumn{2}{|c|}{321} & \multicolumn{2}{|c|}{344} \\
\hline Bic & \multicolumn{2}{|c|}{-24} & \multicolumn{2}{|c|}{-44} \\
\hline \multirow[t]{2}{*}{ Log likelihood } & \multicolumn{2}{|c|}{-1.304} & \multicolumn{2}{|c|}{-1.400} \\
\hline & Coef. & s.e. & Coef. & s.e. \\
\hline age_26 & $-0,272$ & 0,044 & $-0,375$ & 0,113 \\
\hline age_29 & $-0,084$ & 0,032 & $-0,223$ & 0,087 \\
\hline age_32 & $-0,084$ & 0,03 & $-0,112$ & 0,074 \\
\hline age_35 & $-0,112$ & 0,029 & $-0,129$ & 0,067 \\
\hline age_38 & $-0,013$ & 0,028 & $-0,076$ & 0,063 \\
\hline age_41 & $-0,04$ & 0,028 & 0,085 & 0,06 \\
\hline age_44 & $-0,021$ & 0,03 & 0,03 & 0,064 \\
\hline age_47 & $-0,001$ & 0,032 & 0,088 & 0,067 \\
\hline age_50 & 0,024 & 0,034 & 0,035 & 0,076 \\
\hline age_53 & $-0,016$ & 0,038 & 0,136 & 0,087 \\
\hline age_56 & 0,171 & 0,041 & 0,094 & 0,102 \\
\hline age_59 & 0,166 & 0,046 & 0,324 & 0,111 \\
\hline age_62 & 0,282 & 0,051 & 0,121 & 0,113 \\
\hline period_1981 & 0,155 & 0,031 & 0,31 & 0,08 \\
\hline period_1984 & 0,032 & 0,029 & 0,107 & 0,074 \\
\hline period_1987 & 0,108 & 0,027 & 0,21 & 0,064 \\
\hline period_1990 & $-0,03$ & 0,028 & $-0,105$ & 0,067 \\
\hline period_1993 & 0,001 & 0,026 & 0,04 & 0,056 \\
\hline period_1996 & 0,007 & 0,025 & $-0,036$ & 0,057 \\
\hline period_1999 & $-0,047$ & 0,025 & $-0,163$ & 0,058 \\
\hline period_2002 & $-0,132$ & 0,025 & $-0,116$ & 0,057 \\
\hline period_2005 & $-0,095$ & 0,026 & $-0,247$ & 0,064 \\
\hline cohort_1919 & $-0,048$ & 0,206 & 0,57 & 0,614 \\
\hline cohort_1922 & 0,059 & 0,128 & $-0,028$ & 0,593 \\
\hline cohort_1925 & $-0,031$ & 0,101 & $-0,088$ & 0,329 \\
\hline cohort_1928 & 0,15 & 0,084 & $-0,203$ & 0,165 \\
\hline
\end{tabular}




\begin{tabular}{|c|c|c|c|c|}
\hline & \multicolumn{2}{|c|}{ Modelo 1} & \multicolumn{2}{|c|}{ Modelo 2} \\
\hline & \multicolumn{2}{|c|}{$\begin{array}{l}\text { Acesso à universidade } \\
\text { brancos e não brancos }\end{array}$} & \multicolumn{2}{|c|}{$\begin{array}{c}\text { Acesso ao topo da hierarquia ocupacional } \\
\text { brancos e não brancos com diploma } \\
\text { universitário }\end{array}$} \\
\hline \multicolumn{5}{|c|}{ Ajustes do modelo } \\
\hline N & \multicolumn{2}{|c|}{117} & \multicolumn{2}{|c|}{177} \\
\hline g.l. residuais & \multicolumn{2}{|c|}{77} & \multicolumn{2}{|c|}{77} \\
\hline L2 & \multicolumn{2}{|c|}{321} & \multicolumn{2}{|c|}{344} \\
\hline Bic & \multicolumn{2}{|c|}{-24} & \multicolumn{2}{|c|}{-44} \\
\hline \multirow[t]{2}{*}{ Log likelihood } & \multicolumn{2}{|c|}{-1.304} & \multicolumn{2}{|c|}{-1.400} \\
\hline & Coef. & s.e. & Coef. & s.e. \\
\hline cohort_1931 & 0,112 & 0,072 & $-0,212$ & 0,15 \\
\hline cohort_1934 & 0,124 & 0,063 & 0,248 & 0,168 \\
\hline cohort_1937 & 0,145 & 0,055 & 0,091 & 0,152 \\
\hline cohort_1940 & 0,094 & 0,048 & 0,142 & 0,126 \\
\hline cohort_1943 & 0,062 & 0,042 & 0,069 & 0,109 \\
\hline cohort_1946 & 0,035 & 0,038 & 0,234 & 0,093 \\
\hline cohort_1949 & 0,054 & 0,034 & 0,013 & 0,085 \\
\hline cohort_1952 & $-0,004$ & 0,032 & $-0,095$ & 0,078 \\
\hline cohort_1955 & $-0,112$ & 0,031 & $-0,087$ & 0,07 \\
\hline cohort_1958 & $-0,079$ & 0,031 & $-0,18$ & 0,072 \\
\hline cohort_1961 & $-0,092$ & 0,033 & $-0,162$ & 0,072 \\
\hline cohort_1964 & $-0,156$ & 0,036 & $-0,213$ & 0,077 \\
\hline cohort_1967 & $-0,104$ & 0,041 & $-0,008$ & 0,079 \\
\hline cohort_1970 & $-0,074$ & 0,045 & $-0,154$ & 0,098 \\
\hline cohort_1973 & $-0,03$ & 0,051 & 0,009 & 0,107 \\
\hline cohort_1976 & 0,053 & 0,064 & $-0,155$ & 0,138 \\
\hline cohort_1979 & $-0,157$ & 0,117 & 0,211 & 0,215 \\
\hline const. & $-1,198$ & 0,018 & $-1,294$ & 0,056 \\
\hline
\end{tabular}


